\title{
AVALIAÇÃO DO TRATAMENTO DE EFLUENTES CONTENDO RESÍDUOS DE FLUIDOS DE CORTE POR PROCESSO UV- $\mathrm{H}_{2} \mathrm{O}_{2}$
}

\author{
C. ASSENHAIMER ${ }^{1}$, L. N. SETO $^{1}$ e R. GUARDANI ${ }^{1}$ \\ ${ }^{1}$ Universidade de São Paulo, Departamento de Engenharia Química \\ E-mail para contato: cristhianetaka@gmail.com
}

\begin{abstract}
RESUMO - Fluidos de corte são importantes na indústria metal-mecânica por aumentar a vida útil das ferramentas e melhorar a eficiência e qualidade do processo. Em geral, são emulsões de formulações complexas que, ao fim de sua vida útil, geram efluentes que tipicamente contêm água contaminada com níveis de 100 a 30.000 ppm de compostos orgânicos emulsificados. Esses efluentes são de difícil degradação por processos convencionais de tratamento. Uma alternativa consiste nos Processos Oxidativos Avançados. Neste estudo, foi avaliada a eficiência da aplicação do processo UV- $\mathrm{H}_{2} \mathrm{O}_{2}$ no tratamento de um efluente sintético contendo água e resíduos de fluido de corte, por meio de experimentos em laboratório, em diferentes condições. Os resultados indicam redução significativa nos níveis de carbono orgânico dissolvido no efluente e a viabilidade técnica de aplicação do processo UV- $\mathrm{H}_{2} \mathrm{O}_{2}$ no tratamento desses resíduos.
\end{abstract}

\section{INTRODUÇÃO}

Fluidos lubrificantes e óleos de corte desempenham um papel de extrema importância na indústria metal-mecânica. Em processos de usinagem, o uso dos mesmos aumenta a vida útil da ferramenta, minimiza a geração de calor durante o processo, auxilia na remoção de cavacos e melhora a eficiência do sistema produtivo. Em geral, são emulsões de formulações complexas, compostas de uma fase aquosa, uma oleosa, agentes emulsificantes e aditivos químicos diversos, que variam de acordo com o tipo de operação a ser executada e os metais a serem trabalhados.

Uma indústria metal-mecânica típica consome cerca de 33 t/ano de fluido de corte (OLIVEIRA; ALVES, 2007). O consumo mundial anual desses fluidos excede $2 \times 10^{9} \mathrm{~L}$ e o resíduo gerado pode chegar a dez vezes esse valor, visto que os fluidos de corte são diluídos na sua aplicação (CHENG; PHIPPS; ALKHADDAR, 2005). Embora geralmente tenham vida útil longa, quando a substituição por um fluido novo se torna necessária, os fluidos envelhecidos são de difícil disposição, pois tipicamente contêm de 100 a 30.000 ppm de óleo emulsificado e quantidades variáveis de metais pesados (RÍOS; PAZOS; COCA, 1998). Os metais pesados, provenientes de contaminação durante o uso, contribuem ainda mais para a toxicidade e periculosidade desses resíduos. Por essa razão, vários grupos de pesquisa têm proposto soluções para minimização do uso (MACHADO; WALLBANK, 1997) e tratamento dos óleos de corte envelhecidos (GUIMARÃES et al., 2010; HESAMPOUR; KRZYZANIAK; NYSTRÖM, 2008; KOBYA et al., 2008). 


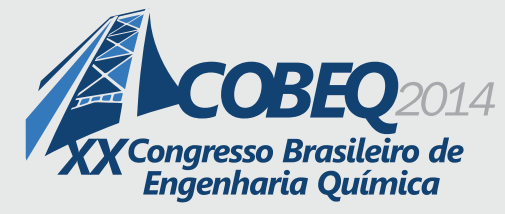

19 a 22 de outubro de 2014

Florianópolis/SC

O tratamento mais comum dado a esses resíduos é a desestabilização química da emulsão, seguida de tratamento biológico, embora diversas outras rotas sejam possíveis (BYERS, 2006). Entretanto, devido à toxicidade e difícil degradação de alguns poluentes, os tratamentos convencionais muitas vezes não são eficientes e a investigação de outras alternativas se torna necessária. Uma opção de tratamento consiste nos Processos Oxidativos Avançados (POA). Ainda que poucos autores tenham estudado a aplicação de POA especificamente para esse tipo de efluente (SEO et al., 2007), esses processos têm se mostrado como uma alternativa técnica com grande potencial para tratamento de diversos poluentes de difícil degradação (LAPERTOT et al., 2007; RIZZO et al., 2008; RUAS, 2008).

Dentre os vários processos oxidativos classificados como POA, o processo baseado na combinação da ação da luz ultravioleta e peróxido de hidrogênio $\left(\mathrm{UV}-\mathrm{H}_{2} \mathrm{O}_{2}\right)$ é o mais antigo (LITTER, 2005). Esse processo ocorre em duas etapas principais: formação de radicais hidroxila pela fotólise direta do $\mathrm{H}_{2} \mathrm{O}_{2}$ (Equação 1) e oxidação das moléculas orgânicas dos contaminantes pelos radicais formados (equações 2, 3 e 4) (BRAUN; OLIVEROS, 1997).

$$
\begin{aligned}
& \mathrm{H}_{2} \mathrm{O}_{2}+h v \rightarrow 2 . \mathrm{OH} \\
& \mathrm{OH}+\mathrm{RH} \rightarrow \mathrm{H}_{2} \mathrm{O}+\mathrm{R} . \\
& . \mathrm{OH}+\mathrm{X}_{2} \mathrm{C}=\mathrm{C}_{2} \mathrm{X} \rightarrow \mathrm{X}_{2} \mathrm{C}(\mathrm{OH})^{-} . \mathrm{CX}_{2} \\
& . \mathrm{OH}+\mathrm{RX} \rightarrow \mathrm{OH}^{-}+\mathrm{XR}^{+} .
\end{aligned}
$$

Uma das vantagens desse método é que não é necessária a adição de outros compostos além do peróxido de hidrogênio, cujo excesso após o fim da reação é degradado naturalmente, não havendo necessidade de etapas posteriores de separação ou outro tipo de tratamento.

Não foram encontrados na literatura até o presente momento outros trabalhos de aplicação desse processo no tratamento de resíduos de fluidos de corte. Porém, o sistema $\mathrm{UV}-\mathrm{H}_{2} \mathrm{O}_{2}$ tem se mostrado eficiente na degradação de diversos poluentes, incluindo resíduos contendo óleos emulsificados (SUBTIL; MIERZWA; HESPANHOL, 2009). Por essa razão, o presente trabalho teve como objetivo avaliar o potencial de aplicação de um sistema $\mathrm{UV}-\mathrm{H}_{2} \mathrm{O}_{2}$ para remoção de contaminantes orgânicos de um efluente sintético contendo resíduos de fluidos de corte, usando medidas de COT como base para avaliação da eficiência do processo.

\section{MATERIAIS E MÉTODOS}

Em todos os experimentos foi utilizado um efluente sintético contendo $0,03 \%$ de fluido de corte, sem a presença de outros interferentes. Essa concentração foi determinada com base em testes anteriores de quebra da emulsão e análise dos resíduos da fase oleosa que permanecem na fase aquosa. O carbono orgânico total (COT) inicial do efluente foi de $186 \pm 20 \mathrm{mg} / \mathrm{L} \mathrm{e} \mathrm{pH} \mathrm{de} \mathrm{7,5.}$

O sistema utilizado nos experimentos está representado na Figura 1. O volume total desse sistema é de $2 \mathrm{~L}$, operado em batelada, com vazão de recirculação de aproximadamente $83 \mathrm{~L} / \mathrm{h}$. Como 
fonte de radiação UV utilizou-se uma lâmpada de vapor de mercúrio de média pressão Philips HPLN de $250 \mathrm{~W}$. Foi verificado em trabalhos anteriores que, para esse sistema, a potência de $250 \mathrm{~W}$ é alta o suficiente para que o fluxo de fótons não seja um fator limitante na reação (LIRA et al., 2006). Para ajuste e correção de $\mathrm{pH}$ foram utilizadas soluções de $\mathrm{NaOH}$ e $\mathrm{H}_{2} \mathrm{SO}_{4}$. A Tabela 1 apresenta os parâmetros operacionais investigados e seus respectivos valores.

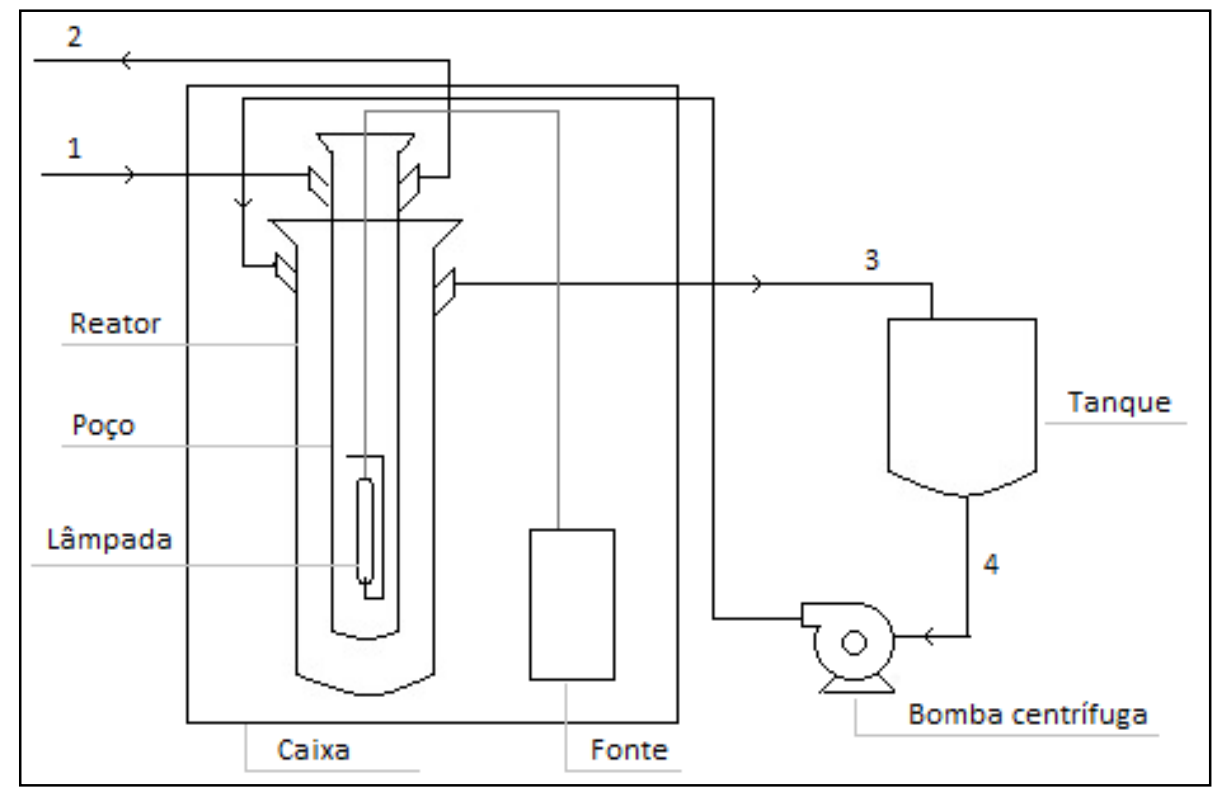

Figura 1 - Representação esquemática do sistema utilizado. As correntes 1 e 2 correspondem à água de resfriamento do sistema e as correntes 3 e 4 correspondem à recirculação do efluente.

Tabela 1 - Parâmetros operacionais investigados

\begin{tabular}{|c|c|}
\hline Parâmetros & Valores \\
\hline Proporção mássica entre COT e $\mathrm{H}_{2} \mathrm{O}_{2}$ & 0,$50 ; 0,94 ; 2,00 ; 3,06 ; 3,50$ \\
\hline $\mathrm{pH}$ & 4,$5 ; 5,4 ; 7,5 ; 9,6 ; 10,5$ \\
\hline
\end{tabular}

Os experimentos foram realizados com base em um planejamento composto central com dois fatores: $\mathrm{pH}$ e proporção em massa entre carbono orgânico dissolvido e peróxido de hidrogênio, tendo como resposta a eficiência de remoção da carga orgânica após 90 min, medida pela redução nos valores de COT pelo analisador TOC/TNM - L, da Shimadzu. Além de valores de pH abaixo do característico do efluente, optou-se por avaliar também valores de $\mathrm{pH}$ superiores, uma vez que, para efluentes mais concentrados, o $\mathrm{pH}$ destes pode ser significativamente maior.

\section{RESULTADOS E DISCUSSÃO}

A Tabela 2 apresenta as condições experimentais, os valores codificados das variáveis do processo $\left(\mathrm{x}_{1}=\right.$ proporção $\mathrm{C}: \mathrm{H}_{2} \mathrm{O}_{2} ; \mathrm{x}_{2}=\mathrm{pH}$ ) e os respectivos resultados da eficiência de remoção de 
COT após 90 min de reação.

Tabela 2 - Condições experimentais e eficiência de remoção de COT para a matriz de experimentos

\begin{tabular}{|c|c|c|c|c|c|}
\hline Exp. & $\mathrm{C}: \mathrm{H}_{2} \mathrm{O}_{2}$ & $p \mathrm{H}$ & $\mathrm{x}_{1}$ & $\mathrm{x}_{2}$ & Eficiência de Remoção de COT (\%) \\
\hline 1 & 0.94 & 5.37 & -1 & -1 & $61.7 \%$ \\
\hline 2 & 3.06 & 5.37 & 1 & -1 & $53.8 \%$ \\
\hline 3 & 0.94 & 9.63 & -1 & 1 & $20.9 \%$ \\
\hline 4 & 3.06 & 9.63 & 1 & 1 & $4.5 \%$ \\
\hline 5 & 0.50 & 7.50 & -1.41 & 0 & $28.2 \%$ \\
\hline 6 & 3.50 & 7.50 & 1.41 & 0 & $19.8 \%$ \\
\hline 7 & 2.00 & 4.50 & 0 & -1.41 & $42.9 \%$ \\
\hline 8 & 2.00 & 10.50 & 0 & 1.41 & $9.5 \%$ \\
\hline 9 & 2.00 & 7.50 & 0 & 0 & $41.6 \%$ \\
\hline 10 & 2.00 & 7.50 & 0 & 0 & $31.8 \%$ \\
\hline 11 & 2.00 & 7.50 & 0 & 0 & $22.6 \%$ \\
\hline
\end{tabular}

Os resultados foram analisados estatisticamente e não foi observada significância da interação entre os fatores ou da proporção entre carbono e peróxido para os valores avaliados; apenas o fator $\mathrm{pH}$ se mostrou significativo. Esse resultado causa estranheza, pois se esperava que variações na quantidade de peróxido adicionado afetassem significativamente o processo. Porém, aparentemente a variabilidade experimental observada na repetição dos pontos centrais é maior que o efeito causado por esse fator, justificando sua baixa significância. Os dados da Tabela 2 também são apresentados na Figura 2.

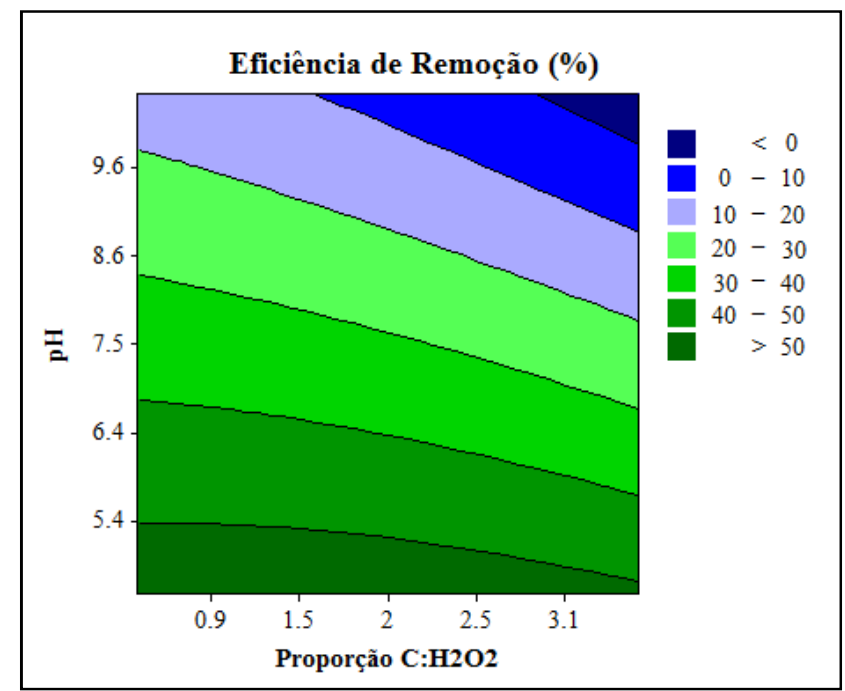

Figura 2 - Efeito dos fatores avaliados na eficiência de remoção de COT.

Observa-se que a redução do pH favorece a remoção de COT do efluente, fornecendo valores de remoção da ordem de $60 \%$. No entanto, avaliando as curvas de remoção com o tempo, exemplificadas 


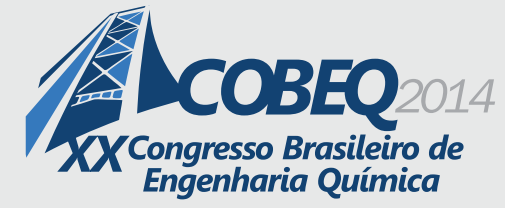

19 a 22 de outubro de 2014

Florianópolis/SC

pelas Figuras 3A, 3B e 3C, é provável que em algumas das condições de processo avaliadas neste estudo possam ser obtidas remoções maiores, se o tempo de experimento for estendido para períodos superiores a $90 \mathrm{~min}$.

Nas Figuras 3B e 3C, percebe-se um comportamento atípico das curvas de remoção de COT e CT com o tempo, em que esses valores aumentam com o tempo até cerca de 60 min e depois caem novamente, indicando que o carbono orgânico dissolvido no efluente aumentou depois desse período de tempo. Embora apenas três das onze curvas obtidas sejam apresentadas aqui, esse comportamento é observado para a maior parte dos experimentos realizados em $\mathrm{pH}$ acima de 7 , sendo mais intenso para valores maiores de $\mathrm{pH}$. Esse comportamento não foi observado em nenhum experimento realizado em pH menor que 7. Esse comportamento deve-se aparentemente ao fato de se tratar de um efluente com carga orgânica emulsificada, ou seja, inicialmente separada da fase aquosa. Neste caso, o processo de degradação consiste primeiramente na destruição das moléculas de emulsificante, seguindo-se da degradação do óleo. Aparentemente, nos experimentos realizados em pH abaixo de 7, a reação foi mais rápida e ocorreu a degradação do emulsificante que estabiliza a emulsão e do óleo emulsificado, gradativamente reduzindo o valor de COT. Nos experimentos em $\mathrm{pH}$ acima de 7, por outro lado, a reação possivelmente é mais lenta, ocorrendo, assim, primeiramente a degradação do emulsificante, o que causa separação de parte do óleo da fase aquosa e redução do COT, medido na fase aquosa. Neste caso, por o óleo estar em concentração muito baixa, a separação deste não pode ser observada visualmente. Na sequência, ocorre a degradação do óleo com a formação de compostos orgânicos mais solúveis em água. Tal fato causa o aumento do valor do COT observado ao final desses experimentos. Um mecanismo semelhante foi encontrado por MORAES et al. (2004) no tratamento de efluentes contendo óleos emulsificados.

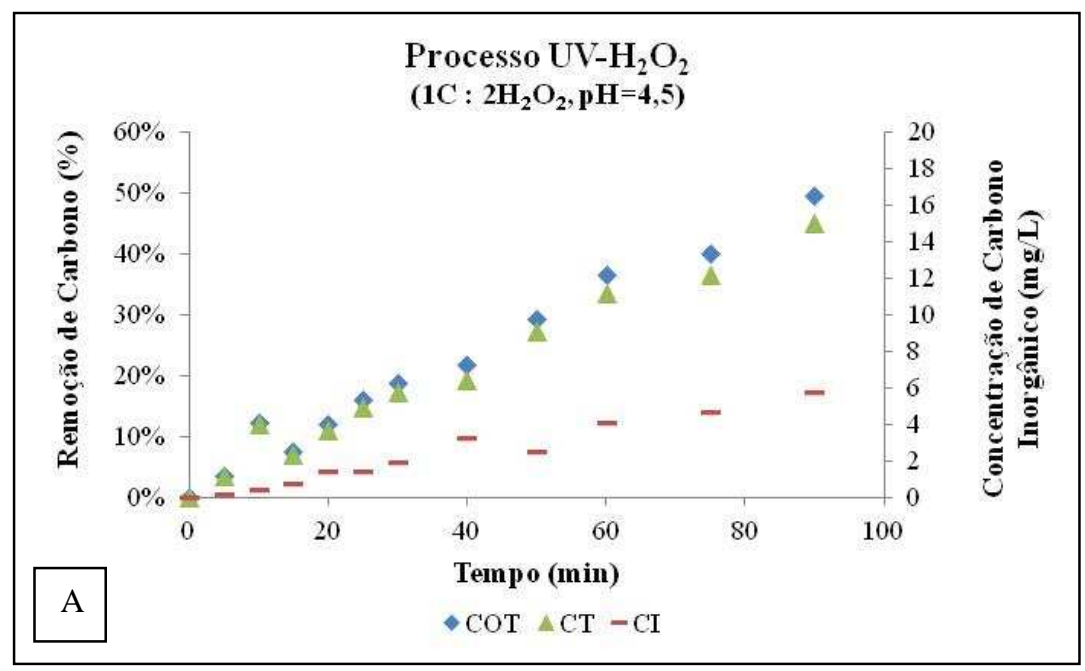




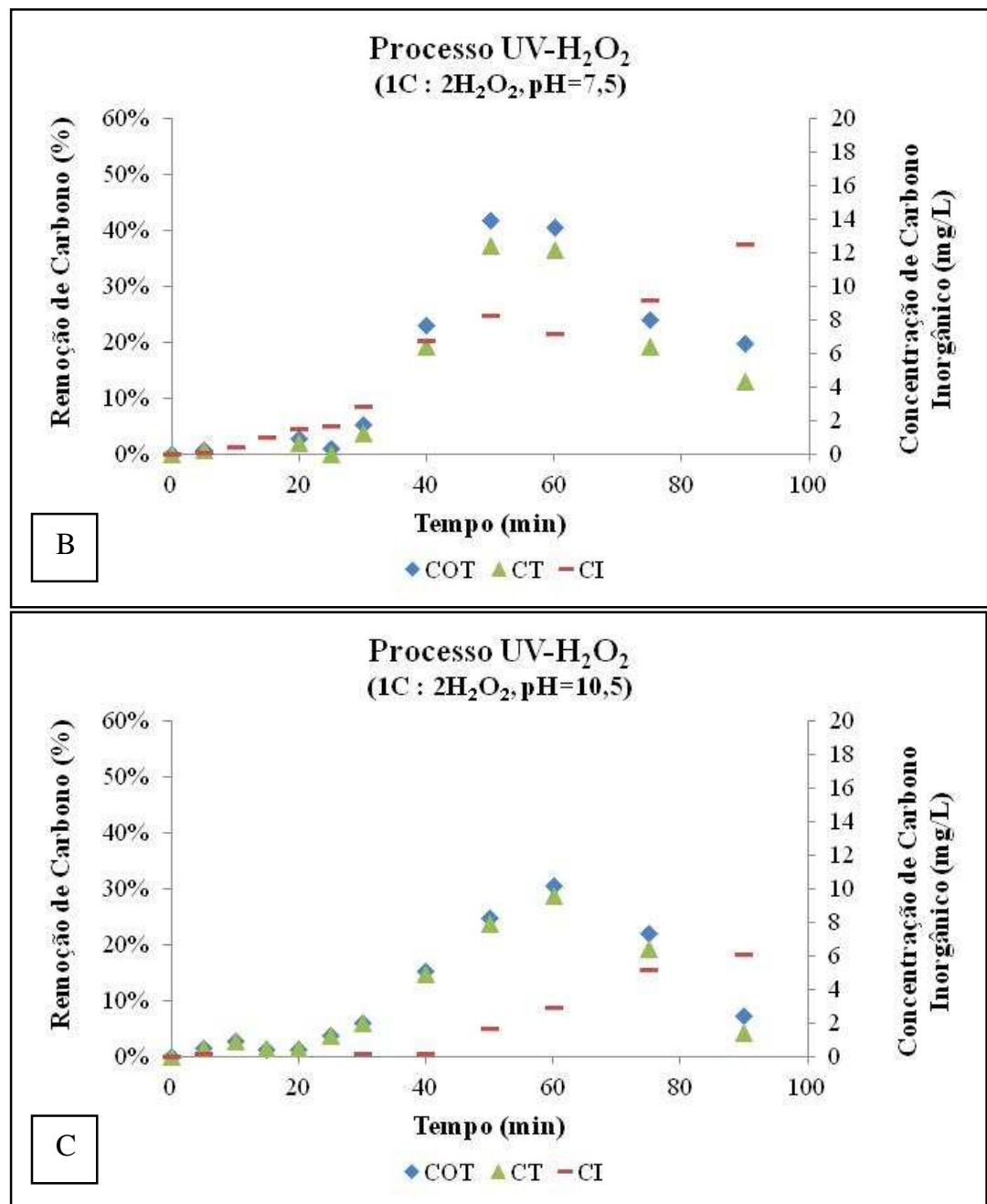

Figura 3 - Exemplo de curvas de remoção de carbono ao longo do tempo, obtidas em diferentes condições de processo $(\mathrm{COT}=$ carbono orgânico total, $\mathrm{CT}=$ carbono total, $\mathrm{CI}=$ carbono inorgânico).

\section{CONCLUSÕES}

A aplicação do processo UV- $\mathrm{H}_{2} \mathrm{O}_{2}$ para tratamento de um efluente sintético contendo resíduos de fluidos de corte apresentou resultados de degradação da carga orgânica da ordem de 60\%, com os melhores resultados obtidos em valores mais baixos de $\mathrm{pH}$. Dentro da faixa analisada, a variação da concentração de peróxido não afetou significativamente o tratamento.

Embora as condições mais adequadas de processo devam ser estimadas a partir de um conjunto maior de experimentos, os resultados apresentados indicam a viabilidade técnica da aplicação do processo $\mathrm{UV}-\mathrm{H}_{2} \mathrm{O}_{2}$ ao tratamento de efluentes provenientes do descarte de emulsões de fluido de corte, constituindo-se, assim, em alternativa a ser considerada visando melhorar a sustentabilidade de processos de manufatura de peças metálicas. 
Um aspecto peculiar do sistema em estudo é o comportamento das curvas de remoção de COT em função do tempo, observado nos experimentos realizados em $\mathrm{pH}$ acima de 7 . Tal fato indica que o tratamento de emulsões por processos de oxidação aparenta ser complexo, por envolver o transporte de massa entre as fases oleosa e aquosa e a transformação de moléculas da fase oleosa em compostos orgânicos solúveis em água.

\section{AGRADECIMENTOS}

Este estudo tem apoio financeiro da FAPESP e foi realizado dentro do escopo de um projeto conjunto entre a Universidade de São Paulo e a Universidade de Bremen, na Alemanha, dentro do programa BRAGECRIM (Brazilian German Cooperative Research Initiative in Manufacturing), financiado pela CAPES e CNPq (Brasil) e DFG (Alemanha).

\section{REFERÊNCIAS}

BRAUN, A.; OLIVEROS, E. How to evaluate photochemical methods for water treatment. Water Sci. and Technol., v. 35, n. 4, p. 17-23, 1997.

BYERS, J. P. Metalworking Fluids. 2a edição. Boca Raton: CRC Press, 2006. p. 480

CHENG, C.; PHIPPS, D.; ALKHADDAR, R. M. Treatment of spent metalworking fluids. Water Res., v. 39, n. 17, p. 4051-63, 2005.

GUIMARÃES, A. P. et al. Destabilization and Recuperability of Oil Used in the Formulation of Concentrated Emulsions and Cutting Fluids. Chem.Biochem.Eng. Q., v. 24, n. 1, p. 43-49, 2010.

HESAMPOUR, M.; KRZYZANIAK, A.; NYSTRÖM, M. Treatment of waste water from metal working by ultrafiltration, considering the effects of operating conditions. Desalination, v. 222, n. 1-3, p. 212-221, 2008.

KOBYA, M. et al. Study on the treatment of waste metal cutting fluids using electrocoagulation. Sep. Purific. Technol., v. 60, n. 3, p. 285-291, 2008.

LAPERTOT, M. et al. Photo-Fenton and biological integrated process for degradation of a mixture of pesticides. J. Photoch. Photobio. A, v. 186, n. 1, p. 34-40, 2007.

LIRA, D. C. B. et al. Estudo para reuso de águas de processos contendo contaminantes. Anais do XVI COBEQ. Santos: 2006. CD-ROM

LITTER, M. I. Introduction to Photochemical Advanced Oxidation Processes for Water Treatment. Hdb. Env. Chem., v. 2, n. M, p. 325-366, 2005. 
MACHADO, A. R.; WALLBANK, J. The effect of extremely low lubricant volumes in machining. Wear, v. 210, n. 1-2, p. 76-82, 1997.

MORAES, J. E. F. et al. Utilization of solar energy in the photodegradation of gasoline in water and of oil-field-produced water. Environ. Sci. Technol., v. 38, n. 13, p. 3746-51, 2004.

OLIVEIRA, J. F. G. DE; ALVES, S. M. Adequação ambiental dos processos usinagem utilizando Produção mais Limpa como estratégia de gestão ambiental. Produção, v. 17, n. 1, p. 129-138, 2007.

RÍOS, G.; PAZOS, C.; COCA, J. Destabilization of cutting oil emulsions using inorganic salts as coagulants. Colloid Surface A, v. 138, n. 2-3, p. 383-389, 1998.

RIZZO, L. et al. Pre-treatment of olive mill wastewater by chitosan coagulation and advanced oxidation processes. Sep. Purific. Technol., v. 63, n. 3, p. 648-653, 2008.

RUAS, D. B. Aplicação do processo oxidativo avançado H2O2/UV como pós-tratamento de reator anaeróbio em efluentes de indústrias de celulose kraft branqueada. São Paulo: Universidade de São Paulo, 2008.

SEO, D. C. et al. Treatment of non-biodegradable cutting oil wastewater by ultrasonicationFenton oxidation process. Water Sci. Technol., v. 55, n. 1-2, p. 251, 2007.

SUBTIL, E.; MIERZWA, J.; HESPANHOL, I. Avaliação do desempenho do sistema UV/H2O2 no tratamento de efluentes provenientes do processo de tratamento térmico de emulsões de água e óleo. Ambiente e Água, v. 4, n. 3, p. 169-180, 312009. 